\title{
Bilateral sagittal split surgery is not a predictable treatment for temporomandibular dysfunction in patients with retrognathia
}

\author{
Kuhlefelt, Marina
}

2016-06

Kuhlefelt , M , Laine , P \& Thoren , H 2016 , ' Bilateral sagittal split surgery is not a predictable treatment for temporomandibular dysfunction in patients with retrognathia ' , Oral surgery, oral medicine, oral pathology and oral radiology , vol. 121 , no. 6 , pp. 595-601 . https://doi.org/10.1016/j.oo

http://hdl.handle.net/10138/224036

https://doi.org/10.1016/j.00oo.2015.12.011

publishedVersion

Downloaded from Helda, University of Helsinki institutional repository.

This is an electronic reprint of the original article.

This reprint may differ from the original in pagination and typographic detail.

Please cite the original version. 


\title{
Bilateral sagittal split surgery is not a predictable treatment @( for temporomandibular dysfunction in patients with retrognathia
}

\author{
Marina Kuhlefelt, DDS, ${ }^{a}$ Pekka Laine, DDS, PhD, ${ }^{b}$ and Hanna Thorén, DDS, MD, PhD ${ }^{c}$
}

Objective. A prospective study to clarify the impact of forward bilateral sagittal split osteotomy (BSSO) on temporomandibular dysfunction (TMD).

Study Design. We examined and interviewed patients with BSSO before and at 1 year after surgery to evaluate the changes in TMD symptoms. A well-known TMD index, which incorporated two complementary subindices-the objective functional Helkimo dysfunction index (Di) and the subjective symptomatic anamnestic index (Ai) - was used. Patients with a forward movement of the mandible and osteosynthesis with titanic miniplates were included.

Results. Forty patients (26 females and 14 males, mean age of study population 36.9 years) retrognathia completed the study. There was no change in TMD symptoms in 24 patients $(60 \%)$, as measured by the $\mathrm{Di}$, and $26(65 \%)$, as measured by the Ai. Twelve patients improved (30\%), according to the Di scores and $10(25 \%)$ according to the Ai scores. Four patients had more TMD symptoms at follow-up (10\%), as measured by both $\mathrm{Di}$ and $\mathrm{Ai}$.

Conclusions. Surgery for orthognathia is a predictable treatment for improving aesthetics and occlusion but less predictable for alleviating TMD symptoms in patients with retrognathia. TMD symptoms should therefore be treated independently. (Oral Surg Oral Med Oral Pathol Oral Radiol 2016;121:595-601)

One of the reasons for retrognathic patients seeking treatment $^{1-3}$ or for dentists referring patients for orthognathic surgery is temporomandibular dysfunction (TMD). Bilateral sagittal split osteotomy ${ }^{4}$ (BSSO) is the standard treatment for the correction of a congenitally small and retrognathic lower jaw. Whether orthognathic surgery is itself a predictable treatment for patients whose primary reason for referral is TMD is a question that needs to be answered.

The question of predictability of TMD in patients with orthognathia has been one of the controversies in oral and maxillofacial surgery, and it is well known that TMD symptoms and clinical findings fluctuate over time. ${ }^{5-7}$ The symptoms can originate from the joint or from the surrounding musculoskeletal structures. Patients often experience pain, joint sounds, muscle tenderness, or deviations or restriction of movement of the mandible.

There is insufficient evidence in the existing published literature, with just a few prospective studies that cover orthognathic surgery and TMD. ${ }^{3,8-12}$ Most studies report favorable corrective results for TMD, even if this includes a subgroup of patients not

\footnotetext{
${ }^{\mathrm{a} C}$ Consultant (Oral and Maxillofacial Surgery), Department of Oral and Maxillofacial Diseases, University of Helsinki, Helsinki, Finland.

${ }^{\mathrm{b}}$ Professor, Department of Oral and Maxillofacial Diseases, University of Helsinki and Helsinki University Hospital, Helsinki, Finland. ${ }^{c}$ Department of Oral and Maxillofacial Diseases, University of Helsinki and Helsinki University Hospital, Helsinki, Finland.

Received for publication Aug 31, 2015; returned for revision Dec 11, 2015; accepted for publication Dec 25, 2015.

(c) 2016 Elsevier Inc. All rights reserved.

2212-4403/\$ - see front matter

http://dx.doi.org/10.1016/j.oooo.2015.12.011
}

benefiting from the treatment. A Finnish study with 82 BSSO patients (64 mandibular advancements and 18 mandibular setbacks) reported that the TMD symptoms were reduced or remained unchanged for $88 \%$ of patients but worsened for $12 \% .^{3}$

In many of the studies, the Helkimo anamnestic index (Ai), the dysfunction index (Di), or slightly modified versions of these were used to evaluate the TMD symptoms. ${ }^{3,9,11}$ Abrahamsson et al. ${ }^{12}$ published a study in 2013 on patients with orthognathia, which used the Research Diagnostic Criteria for Temporomandibular Disorders (RDC/TMD), published in $1992,{ }^{13}$ to evaluate TMD. All previous studies had included different surgical procedures or diagnoses of malocclusions in their final analyses.

There is no previously published prospective study on improving TMD symptoms for patients with mandibular retrognathia undergoing BSSO. We therefore asked the specific question: What is the impact of forward BSSO on TMD symptoms in patients with retrognathia? We then conducted a prospective study with class II patients who were to undergo forward BSSO, with a follow-up time of 1 year, and scored the

\section{Statement of Clinical Relevance}

Our study has shown that the forward movement of a retrognathic mandible with the aid of bilateral sagittal split osteotomy is an unpredictable treatment for temporomandibular dysfunction, although patients often are referred for surgery for these reasons. 
change in TMD symptoms with a widely used TMD index comprising the two aforementioned indicesHelkimo Ai and Di.

\section{PATIENTS AND METHODS}

We designed and carried out a prospective study that included adult patients with Class II mandibular retrognathia. All patients were at least 18 years of age and were to undergo advancement of the mandible with the aid of standard BSSO at the Helsinki University Hospital's Department of Oral and Maxillofacial Diseases, during a period of 18 months. Patients who underwent any other preplanned surgical procedures of the mandible or maxilla during the first postoperative year were excluded. Patients with any other malocclusions or facial syndromes were also excluded, as were patients who had any other surgical procedures in the maxillomandibular region, either before BSSO or during the follow-up, except for patients with surgical complications after BSSO.

All patients underwent preoperative and postoperative orthodontics with fixed orthodontic appliances in both arches to achieve the optimal occlusion. All patients were operated on by an experienced senior surgeon and by a surgeon-in-training. They usually operated on one side each. Fixation using titanium miniplates and monocortical screws was performed in all patients. One horizontal titanium miniplate was placed on each side of the mandible. No other fixation was used, and the patients had no postoperative intermaxillary fixation. All patients had light guiding elastics and a surgical splint for 4 weeks after the operation. A follow-up time of 1 year was required for the patients to be included in the final analysis.

The $\mathrm{Di}$ and the Ai, both of which were published by Helkimo in $1974,{ }^{14}$ were used to register preoperative and 1-year postoperative TMD symptoms. Originally, the TMD index consisted of three different subindices, namely, the clinical dysfunction index (Di), the anamnestic dysfunction index (Ai), and the index for the occlusal state (Oi). The first two, the $\mathrm{Di}$ and the Ai, have since been used in epidemiologic and comparative studies. The Di is based on objective TMD findings during a clinical examination, whereas the $\mathrm{Ai}$ is based on the patient's subjective TMD symptoms and complaints.

The Di is a clinical evaluation of the functional state of the masticatory system, based on the five groups of symptoms (impaired range of movement of the mandible, impaired function of the TMD, pain on movement of the mandible, pain in the temporomandibular joint (TMJ), pain in the masticatory muscles). Every symptom is judged on a three-grade scale of severity: no symptoms awarded 0 points, mild symptoms 1 , and severe symptoms 5. In our study, the scores for the five symptoms were added together. The severity of TMD was scored as follows: clinically symptom-free Di 0 (0 points), mild dysfunction Di I (1-4 points), moderate dysfunction Di II (5-9 points), and severe dysfunction Di III (10-25 points).

The $\mathrm{Ai}$ is based on data obtained by interviewing the patient and is scored on the basis of the TMD symptoms reported by the patient. The patients in our study were divided into three groups (I-III) according to the severity of symptoms reported: patients with no subjective symptoms (Ai 0), patients with mild symptoms of dysfunction (Ai I), and patients with severe symptoms (Ai II). The patients with mild symptoms had one or more of the following symptoms: TMJ sounds, feeling of fatigue of the jaws, and feeling of stiffness of the lower jaw; however, none of the symptoms was described as severe. The patients with severe symptoms (Ai II) reported restrictions in mouth opening, locking, luxation, pain on movement of the mandible, and pain in the TMJ or the masticatory muscles. Facial pain other than pain related to TMD was not included in the index.

TMDs include a wide variety of symptoms of the temporomandibular region, which can be related either to the joints or to the surrounding muscular structures. We used deviations, sounds, locking, and luxation as indicators for joint-related symptoms and the number of muscles tender to palpation as an indicator for musclerelated problems.

All enrolled patients were interviewed and examined according to a standard protocol before and 1 year after the operation by one of the authors (MK). The reason for referral for orthognathic surgery was recorded. We also asked the patients about possible previous TMD and occlusal splints they had. The Helkimo Ai and the Di were recorded.

The study was approved by the Ethics Committee of the Department of Surgery and by the Internal Review Board of the Division of Musculoskeletal Surgery, Helsinki University Central Hospital, Finland. All participating patients signed a written informed consent form. The study followed the principles of the Declaration of Helsinki.

\section{RESULTS}

A total of 42 consecutive patients with retrognathia met the inclusion criteria, and all of the patients consented to participate in the study. Two patients did not appear at the 1-year follow-up, thus their data were excluded, which left the data of the 40 patients who completed the study for the present analysis. Twenty-six patients $(65 \%)$ were female. The mean age of the study population was 36.9 years (range 22.2-59.4 years). The patients were generally healthy. Three patients were taking medication for hypothyreosis, one patient had 
Table I. Helkimo clinical dysfunction index (Di) in 40 patients with bilateral sagittal split before and 1 year after operation

\begin{tabular}{|c|c|c|c|c|c|}
\hline & & Before operation & & One year after operation & \\
\hline Index & Description & No. of patients & $\%$ & No. of patients & $\%$ \\
\hline \multirow[t]{3}{*}{ Mobility index } & 0 (0 points) & 23 & 57.5 & 24 & 60 \\
\hline & 1 (1-4 points) & 14 & 35 & 15 & 37.5 \\
\hline & 5 (5-20 points) & 3 & 7.5 & 1 & 2.5 \\
\hline \multirow[t]{3}{*}{ Temporomandibular function } & No sounds & 28 & 70 & 26 & 65 \\
\hline & Sounds/deviation $>2 \mathrm{~mm}$ & 12 & 30 & 14 & 35 \\
\hline & Locking or luxation & 0 & 0 & 0 & 0 \\
\hline \multirow[t]{3}{*}{ Muscular pain on palpation } & No pain & 18 & 45 & 23 & 57.5 \\
\hline & $1-3$ sites & 11 & 27.5 & 12 & 30 \\
\hline & 4 or more sites & 11 & 27.5 & 5 & 12.5 \\
\hline \multirow[t]{3}{*}{ Joint pain on palpation } & No & 25 & 62.5 & 37 & 92.5 \\
\hline & Lateral aspect pain & 12 & 30 & 3 & 7.5 \\
\hline & Distal aspect pain & 3 & 7.5 & 0 & 0 \\
\hline \multirow[t]{3}{*}{ Pain on mandibular movement } & No & 37 & 92.5 & 37 & 92.5 \\
\hline & One movement & 3 & 7.5 & 2 & 5 \\
\hline & Less than one movement & 0 & 0 & 1 & 2.5 \\
\hline \multicolumn{6}{|l|}{ Clinical dysfunction index (Di) } \\
\hline Clinically symptom free & Di 0 & 11 & 27.5 & 10 & 25 \\
\hline Mild symptoms & Di I & 14 & 35 & 24 & 60 \\
\hline Moderate symptoms & Di II & 13 & 32.5 & 5 & 12.5 \\
\hline Severe symptoms & Di III & 2 & 5 & 1 & 2.5 \\
\hline
\end{tabular}

migraine, and one was obese. Six patients $(15 \%)$ were smokers. All 40 patients had mandibular retrognathia, $33(82.5 \%)$ patients had a deep bite, and $4(10 \%)$ patients had a slight mandibular asymmetry. Four patients were seen to have a slight flattening of the condyle in the preoperative radiographic orthopantomogram, and the condyles appeared normal for the other 36 patients.

Seventeen patients $(42.5 \%)$ had a notation of TMD as one of the reasons for seeking treatment at the first appointment, and 12 patients (30\%) had a history of an occlusal splint for TMD.

The mean operation time was 123 minutes (range 75-165 minutes). Data were missing for one patient. The mean forward movement was $6.3 \mathrm{~mm}$ (range $4.5-10 \mathrm{~mm}$ ). The mandibles of four patients were simultaneously rotated slightly. One patient was reoperated on 2 weeks after the first BSSO, and a refixation was performed because of an unacceptable occlusion.

The preoperative and 1-year postoperative Di results are presented in Table I. At the beginning of treatment, 11 patients had no (27.5\%) TMD, 14 patients had mild (35\%) TMD, and 13 patients had (32.5\%) moderate TMD. Two patients $(5 \%)$ had severe symptoms. Two patients were lost to follow-up and were not included in these numbers. Both had mild symptoms (Di I) at the beginning of treatment. The changes in Di for individual patients are shown in Table II. Four patients developed functional or symptomatic impairments: Three symptom-free patients developed mild symptoms, and in one patient, the symptoms changed from mild to severe. Twelve patients improved their Di or
Table II. Change in Helkimo dysfunction index (Di) for 40 individual patients during follow-up

\begin{tabular}{lcc}
\hline $\begin{array}{c}\text { Di (preoperative)- Di (1 year } \\
\text { postoperative) }\end{array}$ & No. of patients & $\%$ \\
\hline D0-D0 & 8 & 20 \\
D0-DI & 3 & 7.5 \\
DI-D0 & 2 & 5 \\
DI- DI & 11 & 27.5 \\
DI-DIII & 1 & 2.5 \\
DII-DI & 8 & 20 \\
DII-DII & 5 & 12.5 \\
DIII-DI & 2 & 5 \\
& 40 & 100 \\
\hline
\end{tabular}

$\overline{D O}$, clinically symptom free; $D I$, mild symptoms; DII, moderate symptoms; DIII, severe symptoms.

TMD scores: Two patients with mild symptoms became symptom free, and eight patients with moderate symptoms and two patients with initially severe symptoms had mild symptoms at follow-up. Sixteen patients showed no improvement; of these, 11 patients had mild symptoms, and five patients had moderate symptoms at follow-up. Eight patients were symptom free during the whole study. A total of 10 patients were symptom free at follow-up.

Twenty patients $(50 \%)$ had severe symptoms at the beginning of treatment, as evaluated by the $\mathrm{Ai}$ (Table III), and this number decreased to $12(30 \%)$ at the end of the follow-up period. Fifteen (37.5\%) patients were symptom free at the beginning of treatment, and $22(55 \%)$ of the same patients were symptom free 
Table III. Helkimo anamnestic index (Ai) in 40 patients with sagittal split before and 1 year after surgery

\begin{tabular}{|c|c|c|c|c|c|}
\hline$\frac{\text { Index }}{A i}$ & & $\begin{array}{l}\text { Before } \\
\text { surgery }\end{array}$ & $\%$ & $\begin{array}{c}\text { One year } \\
\text { after surgery }\end{array}$ & $\%$ \\
\hline Ai 0 & $\begin{array}{l}\text { Subjectively } \\
\text { symptom-free }\end{array}$ & 15 & 37.5 & 22 & 55 \\
\hline Ai I & Mild symptoms & 5 & 12.5 & 6 & 15 \\
\hline Ai II & Severe symptoms & 20 & 50 & 12 & 30 \\
\hline
\end{tabular}

at the 1-year follow-up. The changes in the Ai score of individual patients are shown in Table IV. The condition of four patients worsened: Three previously symptom-free patients developed severe symptoms, and one with initially mild symptoms ended up with severe symptoms. A total of 10 patients improved; of these, five patients with severe symptoms had mild symptoms, and an additional five patients were symptom free at follow-up. Symptoms were unchanged in 10 patients who had severe symptoms and in four patients with mild symptoms. Twelve patients were symptom free during the whole study. A total of 17 patients were symptom free at the end of the study.

The changes in the Di and the Ai scores during the follow-up period are shown in Table V. The severity of TMD did not change in most patients during the followup period. Ten patients (25\%) had improved Ai scores, and $12(30 \%)$ patients had improved Di scores. The TMD worsened in four (10\%) patients, according to both indices.

Table VI shows the changes in joint- and musclerelated TMD symptoms. Sixteen patients improved or developed impairments during follow-up, as measured by the Di. In seven of the 12 patients who improved, fewer muscles were tender to palpation, whereas the situation remained unchanged for five patients. In one of the four patients who worsened, an increasing number of masticatory muscles were tender to palpation, whereas in the other three, the condition of the masticatory muscles remained unchanged. Only two of the 12 patients who showed improvement had jointrelated symptoms initially; the situation for jointrelated symptoms was unchanged in nine patients, and one patient had more joint-related symptoms. Three of the four patients whose symptoms worsened had more joint-related symptoms, and in one, the situation was unchanged.

\section{DISCUSSION}

The main aim of this study was to clarify the impact of BSSO on patients with retrognathia and TMD. A total of 42 consecutive patients met the inclusion criteria, and all of them agreed to participate in the study. Forty patients completed the 1-year follow-up.
Table IV. Change in Helkimo anamnestic index (Ai) for individual patients during follow-up

\begin{tabular}{lcc}
\hline $\begin{array}{c}\text { Ai (preoperative)- Ai }(1 \text { year } \\
\text { postoperative) }\end{array}$ & No. of patients & $\%$ \\
\hline Ai 0-Ai 0 & 12 & 30 \\
Ai O-Ai II & 3 & 7.5 \\
Ai I-Ai I & 4 & 10 \\
Ai I-Ai II & 1 & 2.5 \\
Ai II-Ai 0 & 5 & 12.5 \\
Ai II-Ai I & 5 & 12.5 \\
Ai II-Ai II & 10 & 25 \\
& 40 & 100 \\
\hline
\end{tabular}

Ai 0 , no symptoms; $A i$, mild symptoms; $A i I I$, severe symptoms.

Table V. Change in temporomandibular dysfunction symptom scores for Ai and Di at 1 year follow-up after bilateral sagittal split osteotomy for 40 patients with sagittal split

\begin{tabular}{lccccc}
\hline & $\begin{array}{c}\text { Anamnestic } \\
\text { index }\end{array}$ & & \multicolumn{2}{c}{$\begin{array}{c}\text { Dysfunction } \\
\text { index }\end{array}$} & \\
\cline { 2 - 2 } Outcome & $\begin{array}{c}\text { No. of } \\
\text { patients }\end{array}$ & $\%$ & $\begin{array}{c}\text { No. of } \\
\text { patients }\end{array}$ & $\%$ \\
\hline $\begin{array}{c}\text { No change in } \\
\text { temporomandibular } \\
\text { symptoms }\end{array}$ & 26 & 65 & 24 & 60 \\
$\begin{array}{l}\text { Improvement of } \\
\text { temporomandibular } \\
\text { symptoms }\end{array}$ & 10 & 25 & 12 & 30 \\
$\begin{array}{l}\text { Impairement of } \\
\text { temporomandibular } \\
\text { symptoms }\end{array}$ & 4 & 10 & 4 & 10 \\
\hline
\end{tabular}

BSSO is probably the most frequently used procedure in the correction of malocclusions. The treatment is lengthy and costly and can have complications. Our data show that one of the main reasons for referrals for BSSO was TMD, and $42.5 \%$ of the study patients had a notation of TMD at the beginning of treatment. The reason for seeking orthognathic treatment reported in a previous Finnish study ${ }^{3}$ was regular headache and facial pain (43\%), TMD problems $(30 \%)$, chewing difficulties (23\%), and dissatisfaction with facial and dental appearance (11\%). There are big cultural differences among countries, and in many parts of the world, aesthetics is the main reason for undergoing orthognathic surgery. ${ }^{15}$ Our study found that 24 $(60 \%)$ patients as measured by the Di and 26 patients $(65 \%)$ as measured by the Ai had no change in their overall TMD scoring. Twelve patients (30\%), according to the Di and 10 patients (25\%) according to the $\mathrm{Ai}$ improved. The TMD condition of four patients $(10 \%)$ worsened regardless of the index used for the evaluation (see Table V).

Most of the existing published reports are divergent in study design and results. A major problem is the lack 
Table VI. Change in joint and muscle TMD symptoms in 16 patients that improved or exacerbated as measured by $\mathrm{Di}$

\begin{tabular}{|c|c|c|c|c|}
\hline & $\begin{array}{l}\text { Muscle pain } \\
\text { by palpation }\end{array}$ & $\begin{array}{l}\text { Muscle pain } \\
\text { by palpation }\end{array}$ & $\begin{array}{c}\text { Joint } \\
\text { dysfunction }\end{array}$ & $\begin{array}{c}\text { Joint } \\
\text { dysfunction }\end{array}$ \\
\hline Symptoms & Di improved & Di impaired & Di improved & Di impairec \\
\hline Improved & 7 & 0 & 2 & 0 \\
\hline Unchanged & 5 & 3 & 9 & 1 \\
\hline Impaired & 0 & 1 & 1 & 3 \\
\hline Total & 12 & 4 & 12 & 4 \\
\hline
\end{tabular}

$T M D$, temporomandibular dysfunction; $D i$, Helkimo dysfunction index.

Joint dysfunction indicates deviation, sound, locking, and luxation.

of an accurate and widely used index for clinical and research purposes, which would make the studies directly comparable and reflect our current knowledge in this field. We chose the complementary Helkimo Di and $\mathrm{Ai}$ in combination as the TMD index, ${ }^{14}$ which was originally published in 1974, because the TMD index gives us the ability to compare our results with those of earlier studies. ${ }^{3,9-11,16-18}$ There are critics of this index, and consequently there has been an effort to create a new and updated index. The main limitations of the index that we used in the present study are that it does not differentiate between muscle-related and jointrelated pathologies, and symptoms that are nowadays considered harmless, such as joint sounds, ${ }^{19}$ are included in the index. Panula ${ }^{9}$ modified the index slightly to be able to categorize patients with muscle, joint, and mixed TMD symptoms, but we chose not to do this because modifying the index even slightly would invalidate any comparison between the data from our patients with those of earlier studies.

One of the main problems with TMD studies is that different pathologies, such as joint and musculoskeletal pathologies, are all regarded as just one combined TMD entity when they could and should be regarded as discrete components. Therefore, we reported the change in muscle-related and joint-related symptoms in Table VI as separate endpoints in an attempt to differentiate between these pathologies. The results indicate that the great majority of the improvements in the Di score were related to alleviation of musclerelated symptoms. In the four patients who were impaired, three patients had an increase in joint-related symptoms. However, no far-reaching conclusions can be drawn from the data of this study.

Another shortcoming is that the Helkimo indices do not consider burden of the TMD condition on the patient on day-to-day basis. Consequently, the "Research Diagnostic Criteria for Temporomandibular Disorders" (RDC/TMD) was published in 1992. ${ }^{13,20}$ This index has been revised to accommodate new research findings that emerged during the years since its inception. ${ }^{21}$ One requirement is that a symptoms index used for research should be in use for an extended period to allow researchers to conduct directly comparable studies of good quality. We found only a few comparable studies $^{12,22}$ on patients with orthognathia actually matching the RDC/TMD.

Previous studies have described highly varying rates of TMD. Our study's Ai scores revealed that $65 \%$ of the patients had no change in TMD symptoms, a further $25 \%$ of the patients improved, and the remaining $10 \%$ had more symptoms 1 year after surgery. The change in symptoms in an individual patient is still unpredictable. Abrahamsson et al. ${ }^{12}$ reported in 2013 that $59.8 \%$ of the patients had no change in their subjective TMD scores after orthognathic surgery, according to the RDC/TMD index. Those authors also reported that $85.4 \%$ of the patients with moderate to severe symptoms at the beginning of the treatment had no or slight symptoms at follow-up. Four patients with no or slight symptoms at the beginning of treatment had moderate or severe symptoms $(10 \%)$ at the end of treatment. Pahkala and Kellokoski ${ }^{3}$ found that a significant proportion of the patients benefited from the treatment, even when $12 \%$ of their patients with TMD worsened after BSSO. A study conducted in 2002 by Dervis and Tuncer ${ }^{10}$ found that $10 \%$ of the patients developed new TMD symptoms, which is in line with the findings of our present study.

It is well known that TMD symptoms vary widely over time, and therefore Abrahamsson et al. ${ }^{12}$ also compared the results in the orthognathia group with a control group consisting of patients referred for normal dental treatment and occlusion, with no need for orthodontics or orthognathic surgery. Abrahamsson et al. found that $78.9 \%$ had no change in symptoms, $60 \%$ of the patients with moderate to severe symptoms improved, and $15.5 \%$ of the patients with no or slight symptoms at the beginning of the follow-up period had moderate to severe symptoms at the end of the follow-up period. This indicates that there was actually only a very small difference between the patients undergoing BSSO and the natural course of TMD in the control group. Onizawa et al. ${ }^{8}$ came to the same conclusion in 1995 in their prospective study on 30 patients with orthognathia and 30 healthy volunteers, and they suggested that alterations of TMD after orthognathic surgery do not always result from the actual surgical correction of the malocclusion. We also agree with this conclusion. There are no randomized, doubleblinded studies that have investigated TMD in patients with orthognathia. This might partly be explained by the fact that it would be unethical to postpone surgery for some patients with a severe malocclusion, just for research purposes. 
The correct position of the condylar head is of the utmost importance in BSSO patients. There has been discussion about the different types of osteosynthesis and their impact on TMD. We used titanium miniplates and monocortical screws. Their fixation was semi-rigid, which allows small adaptive changes to occlusion and the condyles during function. Screw fixation is rigid and unforgiving, and there is a risk for condylar torque, at least in the hands of an inexperienced surgeon. There is scant evidence in the literature on this topic. Yamashita et al. ${ }^{18}$ showed that patients with a mandibular setback and mini-plate fixation had significantly less severe TMD symptoms compared with patients who had undergone fixation with bicortical screws immediately after the operation and during a follow-up period of 5 years. They concluded that patients with plate fixation tended to recover faster and had fewer symptoms of TMD.

\section{CONCLUSIONS}

Orthognathic treatment is lengthy and costly and can have complications. Its efficacy for improving TDM symptoms is still controversial. The outcome with regard to the alleviation of TMD symptoms is still unpredictable, and in the light of our current knowledge, there is no sure way to predict the final result of TMD in an individual patient. Patients and referring colleagues should get appropriate information about this lack of certainty, to reduce unrealistic expectations and thereby circumvent patient dissatisfaction after treatment. We agree with the conclusion of Nadershah and Mehra ${ }^{23}$ that occlusion and TMD should be treated as two separate entities. Most patients are highly satisfied with the treatment, and the improvement of occlusion and facial aesthetics is unquestionable.

There is an urgent need for more studies that use a well-known TMD index with a well-defined follow-up time and a robust study design. TMD symptoms in individual patients fluctuate over time, and thus studies with multiple measuring points, better differentiation among TMD pathologies, and a longer follow-up time would improve our current understanding of TMD.

\section{REFERENCES}

1. Forssell H, Finne K, Panula K, Blinikka L-M. Expectations and perceptions regarding treatment: a prospective study of patients undergoing orthognathic surgery. Int J Adult Orthod Orthognath Surg. 1998;13:107-113.

2. Nurminen L, Pietilä T, Vinkka-Puhakka H. Motivation for and satisfaction with orthodontic-surgical treatment: a retrospective study of 28 patients. Eur J Orthod. 1999;21:79-87.

3. Pahkala R, Kellokoski J. Surgical-orthodontic treatment and patient's functional and psychosocial well-being. Am J Orthod Dentofacial Orthop. 2007;132:158-164.
4. Patel P, Novia M. The surgical tools: the Le Fort I, bilateral sagittal split osteotomy of the mandible, and the osseous genioplasty. Clin Plast Surg. 2007;34:447-475.

5. Magnusson T, Egermark I, Carlsson GE. A Longitudinal epidemiologic study of signs and symptoms of temporomandibular disorders from 15 to 35 years of age. J Orofac Pain. 2000; $14: 310-319$

6. Magnusson T, Egermark I, Carlsson GE. A prospective investigation over two decades on signs and symptoms of temporomandibular disorders and associated variables. A Final summary. Acta Odontol Scand. 2005;63:99-109.

7. Scrivani S, Keith D, Kaban L. Temporomandibular disorders. $N$ Engl J Med. 2008;359:2693-2705.

8. Onizawa K, Schmelzeisen R, Vogt S. Alteration of Temporomandibular joint symptoms after orthognathic surgery. J Oral Maxillofacial Surg. 1995;53:117-121.

9. Panula K, Somppi M, Finne K, Oikarinen K. Effects of orthognathic surgery on temporomandibular joint dysfunction. A controlled prospective 4-year follow-up study. Int J Maxillofac Surg. 2000;29:183-187.

10. Dervis E, Tuncer E. Long-term evaluations of temporomandibular disorders in patients undergoing orthognathic surgery compared with a control group. Oral Surg Oral Med Oral Pathol Oral Radiol Endod. 2002;94:554-560.

11. Pahkala R, Heino J. Effects of sagittal split ramus osteotomy on temporomandibular disorders in seventy-two patients. Acta Odontol Scand. 2004;62:238-244.

12. Abrahamsson C, Henriksson M, Nilner B, Sunzel L, Bondemark E. TMD before and after correction of dentofacial deformities by orthodontic and orthognathic treatment. Int J Oral Maxillofac Surg. 2013;42:752-758.

13. Dworkin S, LeResche L. Research diagnostic criteria for temporomandibular disorders: review, criteria, examinations and specifications, critique. J Craniomandib Disord. 1992;6:301-355.

14. Helkimo M. Studies on function and dysfunction of the masticatory system. II. Index for anamnestic and clinical dysfunction and occlusal state. Sven Tandlak Tidskr. 1974;67:101-119.

15. Yu D, Wang F, Wang X, Fang B, Shen S. Presurgical motivations, self-esteem, and oral health of orthognathic surgery patients. J Craniofac Surg. 2013;24:743-747.

16. Yamashita Y, Mizuashi K, Shigematsu M, Goto M. Masticatory function and neurosensory disturbance after mandibular correction by bilateral sagittal split ramus osteotomy: a comparison between miniplates and bicortical screw rigid internal fixation. Int J Oral Maxillofac Surg. 2007;36:118-122.

17. Bock J, Maurer P, Fuhrmann R. The importance of temporomandibular function for patient satisfaction following orthognathic surgery. J Orofac Orthop. 2007;68:299-307.

18. Yamashita Y, Otsuka T, Shigematsu M, Goto M. A long-term comparative study of two rigid internal fixation techniques in terms of masticatory function and neurosensory disturbance after mandibular correction by bilateral sagittal split ramus osteotomy. Int J Oral Maxillofac Surg. 2011;40:360-365.

19. Naeije M, Veldhuis A, Veldhuis E, Visscher C, Lobbezoo F. Disc displacement within the human temporomandibular joint: a systemic review of a 'noisy annoyance. J Oral Rehabil. 2013;40:139-158.

20. Truelove E, Sommers E, LeResche L, Dworkin S, von Korff M. Clinical diagnostic criteria for TMD. New classification permits multiple diagnoses. J Am Dent Assoc. 1992;123:47-54.

21. Schiffman E, Ohrbach R, Truelove E, et al. The revised research diagnostic criteria for temporomandibular disorders: methods used to establish and validate revised Axis I Diagnostic algorithms. J Orofac Pain. 2010;24:63-78. 
22. Farella M, Michelotti A, Boccino T, Cimino R, Laino A, Steenks M. Effects of orthognathic surgery for class III malocclusion on signs and symptoms of temporomandibular disorders and on pressure pain thresholds of the jaw muscles. Int $J$ Oral Maxillofac Surg. 2007;36:583-587.

23. Nadershah M, Mehra P. Orthognathic surgery in the presence of temporomandibular dysfunction: what happens next? Oral Maxillofacial Surg Clin N Am. 2015;27:11-26.
Reprint requests:

Marina Kuhlefelt, DDS

consultant (Oral and Maxillofacial Surgery)

Department of Oral and Maxillofacial Diseases

University of Helsinki

Helsinki, Finland

Marina@hammasfreda.com; marina.kuhlefelt@helsinki.fi 